\title{
The Prevalence and Risk Factors for S2 Alar-Iliac Screw Loosening with a Minimum 2-Year Follow-up
}

\author{
Hiroaki Nakashima ${ }^{1}$, Tokumi Kanemura ${ }^{1}$, Kotaro Satake $^{1}$, Kenyu Ito $^{1}$, Yoshimoto Ishikawa ${ }^{1}$, \\ Jun Ouchida ${ }^{1}$, Naoki Segi ${ }^{2}$, Hidetoshi Yamaguchi ${ }^{2}$, Shiro Imagama ${ }^{2}$ \\ ${ }^{1}$ Department of Orthopedic Surgery, Konan Kosei Hospital, Konan, Japan \\ ${ }^{2}$ Department of Orthopedic Surgery, Nagoya University Graduate School of Medicine, Nagoya, Japan
}

\begin{abstract}
Study Design: A retrospective cohort study.
Purpose: The purpose of this study was to investigate the prevalence and risk factors for S2 alar-iliac (SAl) screw loosening following lumbosacral fixation, with a minimum 2-year follow-up.

Overview of Literature: Although SAI screws allow surgeons to perform lumbosacral fixation with a low profile and enhanced biomechanical strength, screw loosening following surgery can occur in some cases. However, few studies have investigated the prevalence and risk factors for SAl screw loosening.

Methods: This retrospective study included 35 patients (mean age, $72.8 \pm 8.0$ years; male, 10; female, 25 ) who underwent lumbosacral fixation using SAl screws with at least 2 years of follow-up. SAl screw loosening and L5-S bony fusion were assessed using computed tomography. The period for which the screws appeared loose and the risk factors for SAl screw loosening were investigated 2 years after surgery.

Results: A total of 70 SAI screws and 70 S1 pedicle screws were inserted. Loosening was observed 0.5, 1, and 2 years after surgery in $17(24.3 \%), 35(50.0 \%)$, and $35(50.0 \%)$ SAl screws, respectively. Bony fusion rate at L5-S was significantly lower in patients with SAl screw loosening than in those without screw loosening (65.0\% vs. 93.3\%, $p=0.048)$. The score for SAl screw contact with the iliac cortical bone and the bony fusion rate at $\mathrm{L} 5-\mathrm{S}$ were significantly lower in the loosening group than in the non-loosening group (1.8 \pm 0.5 vs. $2.2 \pm 0.3, p<0.001$, respectively). Postoperative pelvic incidence-lumbar lordosis was significantly higher in the loosening group than in the non-loosening group $\left(7.9^{\circ} \pm 15.4^{\circ}\right.$ vs. $0.5^{\circ} \pm 8.7^{\circ}, p=0.02$, respectively).

Conclusions: SAl screw loosening is closely correlated with pseudoarthrosis at L5-S. Appropriate screw insertion and optimal lumbar lordosis restoration are important to prevent postoperative complications related to SAI screws.
\end{abstract}

Keywords: S2 alar-iliac screw; Bony fusion; Screw loosening; Risk factors

\section{Introduction}

Rigid distal fixation of the spine is essential for maintaining proper sagittal and coronal balance. Lumbosacral fusion provides a biomechanical support at the base of a long construct; however, high instrument failure and reoperation rates have been reported when $\mathrm{S} 1$ screws are used alone [1,2]. The L5-S junction has the highest risk

Received Apr 30, 2019; Revised Jun 22, 2019; Accepted Jun 25, 2019

Corresponding author: Hiroaki Nakashima

Department of Orthopedic Surgery, Konan Kosei Hospital, 137 Takayamachi Omatsubara, Konan 483-8704, Japan

Tel: +81-587-51-3333, Fax: +81-587-51-3300, E-mail: hirospine@gmail.com 
of pseudoarthrosis, with a reported rate of $24 \%$ and is associated with adverse clinical outcomes [3]. Multiple studies have shown that long instrumentation and fusion at the sacrum require supplemental pelvic fixation in order to prevent these complications [1,2]. To date, several techniques have been reported, including the use of iliac screws, S2 pedicle screws [4], sacral alar screws [5], and S2 alar-iliac (SAI) screws [1,6]. Among these techniques, the use of SAI screws is a very effective approach using supplemental instrumentation, because SAI screws do not require offset connectors, have a low profile, and can be aligned with cephalad instrumentation, which can minimize wound breakdown, surgical time, possible infection risk, and morbidity [1,6-8].

Although favorable outcomes have been reported with the use of SAI screws in adult spinal deformity surgery, SAI screw loosening or pseudoarthrosis at L5-S junction has been reported in some cases. Screw loosening, observed as a radiolucent area around the screw, indicates loss of screw fixation and potential progression to pseudoarthrosis. Few studies have investigated the prevalence and risk factors for SAI screw loosening; therefore, limited information is available on this subject. The risk factors for distal junctional failure after long instrument fusion using iliac screws include advanced age, low screw density, large pelvic incidence (PI), revision surgery, failure to restore lumbar lordosis (LL), postoperative sagittal imbalance, and insufficient sacropelvic fixation [3,9-11]. However, it is unclear whether these risk factors are associated with SAI screw loosening. Therefore, this study aimed to investigate the prevalence and risk factors for SAI screw loosening following lumbosacral fixation.

\section{Materials and Methods}

\section{Study design}

After approval by the relevant institutional ethics committee (2019-006 [0339]), we retrospectively reviewed the radiographic records of patients who had undergone bilateral lumbosacral fusion with SAI screws between 2013 and 2015. In the current study, three attending spine surgeons performed the surgeries in one hospital. We excluded patients who met any of the following criteria: age $<20$ years, number of fusion levels $<4,<2$ years of followup, no L5-S posterior (PLIF) or transforaminal lumbar interbody fusion (TLIF), and pelvic fixation performed with $>2$ SAI screws.

\section{Radiographic analysis}

Computed tomography (CT) and radiographs were taken at preoperative, 6 months, 1 year, and 2 years after surgery for all patients. Both SAI and S1 screw loosening were assessed using CT reconstruction images. Screw loosening was considered if a radiolucent area ( $>1 \mathrm{~mm}$ in width) was present around the screw (Fig. 1). Radiological bony fusion at L5-S was also evaluated using CT reconstruction images. We judged whether body fusion had been achieved in cases with continuous bony bridging on sagittal and coronal CT scans.

Radiographic assessment was performed by two independent spinal surgeons who did not perform any surgeries in the current case series. A third reviewer was available for adjudication in case of disagreement.

The relationship between SAI screws and iliac bone was assessed using X-rays and CT (Fig. 2). The degree of contact was graded according to the number of contact points between the SAI screw and the iliac cortical bone (range, $0-3$ ): grade 3, 3 contact points (screw contact with the inner, outer, and inferior walls of the iliac cortical bone); grade 2, 2 contact points ( 2 out of 3 contact points involving the iliac cortical bone); grade 1, 1 contact point (1 out of 3 contact points involving the iliac cortical bone); and grade 0,0 contact points (no contact between the screw and the iliac cortical bone).

Preoperative and immediately postoperative (first instance of standing) whole-spine images were obtained in an upright standing position. The following radiological parameters were assessed: LL, pelvic tilt (PT), PI, C7, sag-

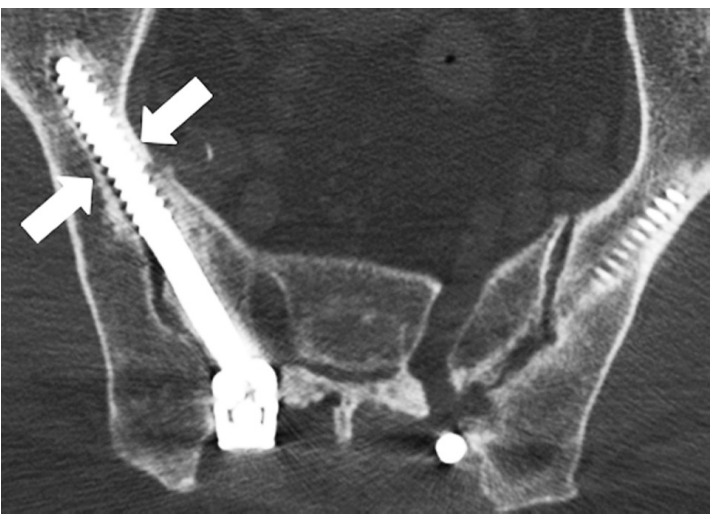

Fig. 1. Assessment of the radiolucent area (white arrows) around S2 alar-iliac screws on computed tomography. 

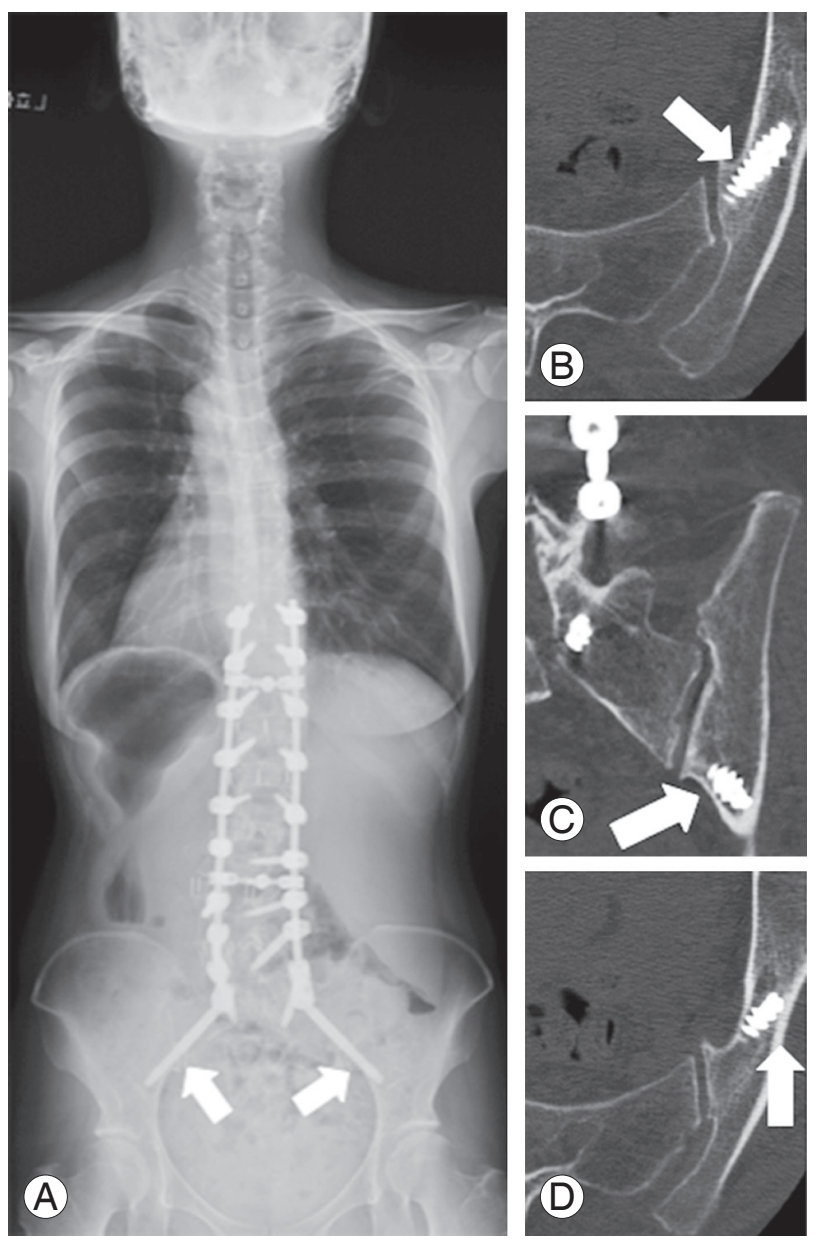

Fig. 2. Assessment of the number of $S 2$ alar-iliac screw contact points with the iliac cortical bone on X-ray (A) and CT (B-D). When the screw came in contact with the inner, outer, and inferior walls of the iliac cortical bone on CT (white arrows), the number of contacts noted were 3. CT, computed tomography.

ittal vertical axis, thoracic kyphosis, and $\mathrm{T} 1$ pelvic angle.

\section{Surgical procedure}

The PLIF or TLIF technique was used for interbody fusion at L5-S1 in all patients. Two carbon fiber interbody cages filled with local bone (obtained during posterior decompressive laminotomy by removing all soft tissues) were inserted. The bone graft was packed around and between the cages as much as possible.

SAI screws were placed using fluoroscopic guidance or the Medtronic Stealth Station Treon with O-arm (Medtronic Inc., Littleton, MA, USA) navigation guidance (Fig. 3). The starting point was $2-3 \mathrm{~mm}$ inferior to the $\mathrm{S}-1$ dorsal foramen and 2-3 mm lateral to it. When inserting SAI screws using fluoroscopic guidance, anteroposterior
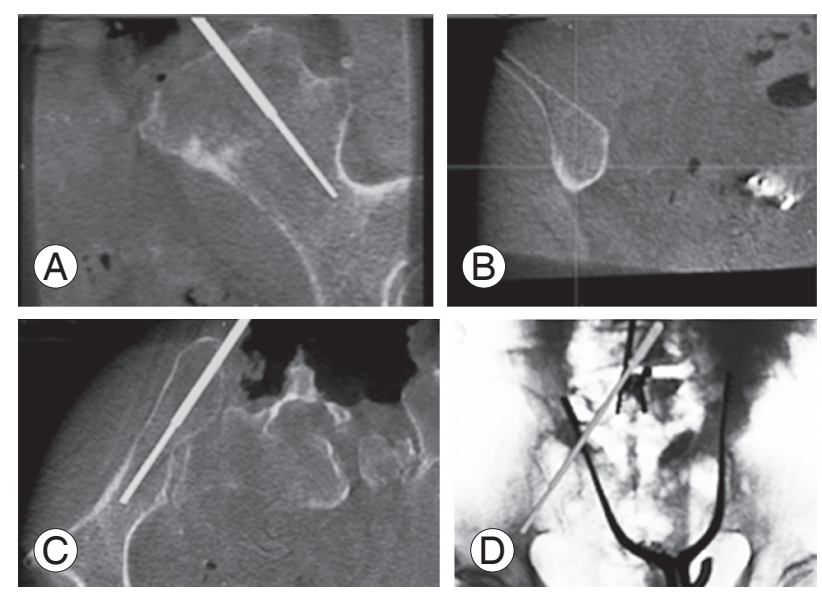

Fig. 3. (A-D) A representative three-dimensional guidance system image for S2 alar-iliac screw insertion.

$\mathrm{X}$-rays were used to ensure placement cephalad to the sciatic notch. A pelvic inlet radiograph was then used to ensure extrapelvic placement, which was assessed by viewing the anterior sacroiliac joint. Angulation was directed toward the greater trochanter and was approximately $30^{\circ}$ anterior from the floor. All patients received posterior bone grafts using local bone, with L5-S interbody fusion.

\section{Statistical analysis}

Data are presented as mean \pm standard deviation. Student $t$-test and the chi-square test were used for comparisons. All statistical analyses were performed using IBM SPSS ver. 21.0 (IBM Corp., Armonk, NY, USA). A $p$-value $<0.05$ was considered statistically significant.

\section{Results}

This study included 35 patients (mean age, $72.8 \pm 8.0$ years; male, 10; female, 25). The mean number of fusion levels was $7.7 \pm 3.0$. Primary diagnosis was adult spinal deformity and adjacent segment disease in 28 and seven patients, respectively. Mean follow-up period after surgery was $31.8 \pm 5.5$ months. Fluoroscopic guidance and the Medtronic Stealth Station Treon with O-arm navigation guidance were used in $2(5.7 \%)$ and $33(94.3 \%)$ patients, respectively.

A total of $70 \mathrm{SAI}$ screws and $70 \mathrm{~S} 1$ pedicle screws were inserted. Loosening was observed in 13 (18.6\%), 13 (18.6\%), and $11(15.7 \%)$ S1 pedicle screws at $0.5,1$, and 2 years after surgery, respectively. L5-S interbody fusion was observed in 7 (20.0\%), 16 (45.7\%), and 27 (77.1\%) 
cases at $0.5,1$, and 2 years after surgery, respectively. One patient with pseudoarthrosis at L5-S underwent revision surgery owing to rod breakage between L5 and S1.

Penetration of a single SAI screw through the iliac cortical bone was observed on postoperative CT. This screw was inserted with assistance from the $\mathrm{O}$-arm navigation system. Although the screw penetrated to a point which was $2 \mathrm{~mm}$ from the lateral cortex of the iliac bone, there were no clinical symptoms attributable to this penetration.

Loosening was observed in 17 (24.3\%), 35 (50.0\%), and 35 (50.0\%) SAI screws at $0.5,1$, and 2 years after surgery, respectively. SAI screw loosening at 2 years was observed in 20 patients (57.1\%), with unilateral loosening in five patients and bilateral loosening in 15 patients. The score for contact between the SAI screw and the iliac cortical bone and the bony fusion rate at L5-S were significantly lower in the loosening group than in the non-loosening group (1.8 \pm 0.5 versus $2.2 \pm 0.3, p<0.001$ and $65.0 \%$ versus $93.3 \%, p=0.048$, respectively) (Table 1 ). However, the groups did not differ significantly in terms of age, sex, bone mineral density (BMD), upper instrumented vertebrae, number of fusion levels, insufficient correction (PILL $>10^{\circ}$ ), and S1 pedicle screw loosening (Table 1). With regard to radiographic parameters, preoperative PT and postoperative PI-LL were significantly higher in the loosening group than in the non-loosening group $\left(22.2^{\circ} \pm 13.3^{\circ}\right.$ versus $28.6^{\circ} \pm 9.3^{\circ}, p=0.02$ and $7.9^{\circ} \pm 15.4^{\circ}$ versus $0.5^{\circ} \pm 8.7^{\circ}$,

Table 1. Risk factors of postoperative SAl screw loosening in the study patients

\begin{tabular}{|c|c|c|c|}
\hline \multirow{2}{*}{ Characteristic } & \multicolumn{2}{|c|}{ SAI screw loosening } & \multirow{2}{*}{$p$-value } \\
\hline & Yes & No & \\
\hline No. of patients & 20 & 15 & \\
\hline Age (yr) & $73.9 \pm 7.9$ & $71.7 \pm 8.0$ & 0.28 \\
\hline Gender & & & 0.19 \\
\hline Male & 4 & 6 & \\
\hline Female & 16 & 9 & \\
\hline Bone mineral density $\left(\mathrm{mg} / \mathrm{cm}^{2}\right)$ & $1.16 \pm 0.3$ & $1.23 \pm 0.3$ & 0.41 \\
\hline Upper instrumented vertebra & & & 0.12 \\
\hline Above T6 & 3 & 0 & \\
\hline Below T6 & 17 & 15 & \\
\hline No. of fusion levels & $8.0 \pm 3.2$ & $7.3 \pm 2.7$ & 0.31 \\
\hline No. of interbody fusion levels above L5-S1 & $3.3 \pm 1.0$ & $3.5 \pm 0.9$ & 0.55 \\
\hline No. of SAI screw contact points with the iliac cortical bone & $1.8 \pm 0.5$ & $2.2 \pm 0.3$ & $<0.001^{*}$ \\
\hline Insufficient correction (pelvic incidence-lumbar lordosis $>10^{\circ}$ ) & & & 0.15 \\
\hline Yea & 7 & 2 & \\
\hline No & 13 & 13 & \\
\hline L5-S fusion at 2 years & & & $0.048^{*}$ \\
\hline Yes & 13 & 14 & \\
\hline No & 7 & 1 & \\
\hline S1 pedicle screw loosening at 2 years & & & 0.15 \\
\hline Yes & 5 & 1 & \\
\hline No & 15 & 14 & \\
\hline Rod breakage at L5-S1 & & & 1.00 \\
\hline Yes & 1 & 0 & \\
\hline No & 19 & 15 & \\
\hline
\end{tabular}

Values are presented as number or mean \pm standard deviation, unless otherwise stated.

SAI, S2 alar-iliac.

" $p<0.05$; statistically significant. 
$p=0.02$, respectively) (Table 2 ).

\section{Discussion}

The present study found that the rate of SAI screw loosening was $50.0 \%$ at one and 2 years after surgery and that screw loosening was correlated with pseudoarthrosis at L5-S on CT. In addition, a low number of SAI screw contact points with the iliac cortical bone and postoperative PI-LL $>10^{\circ}$ were significant risk factors for screw loosening, indicating that appropriate screw insertion and optimal LL restoration are crucial for preventing postoperative complications.

Lumbosacral fixation is still a challenge for spinal surgeons when trying to achieve solid fusion. Although many supplemental pelvic fixation techniques have been reported, the use of SAI screws is one of the most effective approaches for the following reasons: (1) the screws are in line with the rest of the instrumentation, rendering offset connectors unnecessary; (2) soft tissue dissection is minimized, especially in the paraspinal muscles around the iliac crest; (3) wound breakdown is minimized because

Table 2. Comparison of radiographic parameters between the SAI screw loosening and non-loosening groups

\begin{tabular}{|c|c|c|c|}
\hline \multirow{2}{*}{ Variable } & \multicolumn{2}{|c|}{ SAI screw loosening } & \multirow{2}{*}{$p$-value } \\
\hline & Yes & No & \\
\hline No. of patients & 20 & 15 & \\
\hline \multicolumn{4}{|l|}{ Preoperative } \\
\hline $\operatorname{Pl}\left({ }^{\circ}\right)$ & $55.7 \pm 11.0$ & $51.7 \pm 6.0$ & 0.07 \\
\hline $\operatorname{LL}\left({ }^{\circ}\right)$ & $23.5 \pm 18.6$ & $19.9 \pm 16.4$ & 0.40 \\
\hline PI-LL ( $\left(^{\circ}\right)$ & $32.2 \pm 18.8$ & $31.8 \pm 18.3$ & 0.93 \\
\hline $\operatorname{PT}\left({ }^{\circ}\right)$ & $22.2 \pm 13.3$ & $28.6 \pm 9.3$ & $0.02^{*}$ \\
\hline SVA (mm) & $85.1 \pm 40.8$ & $93.9 \pm 56.8$ & 0.46 \\
\hline $\operatorname{TPA}\left({ }^{\circ}\right)$ & $22.7 \pm 12.6$ & $27.7 \pm 12.0$ & 0.10 \\
\hline \multicolumn{4}{|l|}{ Postoperative } \\
\hline $\operatorname{LL}\left({ }^{\circ}\right)$ & $47.9 \pm 13.1$ & $51.3 \pm 10.1$ & 0.23 \\
\hline $\mathrm{PI}-\mathrm{LL}\left({ }^{\circ}\right)$ & $7.9 \pm 15.4$ & $0.5 \pm 8.7$ & $0.02^{*}$ \\
\hline $\operatorname{PT}\left({ }^{\circ}\right)$ & $18.8 \pm 10.5$ & $18.7 \pm 8.0$ & 0.96 \\
\hline SVA (mm) & $33.9 \pm 47.5$ & $21.5 \pm 26.3$ & 0.19 \\
\hline $\operatorname{TPA}\left({ }^{\circ}\right)$ & $20.6 \pm 11.9$ & $18.3 \pm 7.2$ & 0.34 \\
\hline
\end{tabular}

Values are presented as number or mean \pm standard deviation, unless otherwise stated.

SAI, S2 alar-iliac; PI, pelvic incidence; LL, lumbar lordosis; PT, pelvic tilt; SVA, C7 sagittal vertical axis; TPA, T1 pelvic angle.

" $p<0.05$; statistically significant. the SAI screw anchors are placed approximately $15 \mathrm{~mm}$ deeper than with iliac screws; and (4) a biomechanically strong anchor is achieved because the screws extend beyond the lumbosacral pivot point and the screw trajectories are directed more laterally, which helps reduce screw back-out $[1,7,8]$.

Many reports on SAI screws have discussed favorable outcomes related to deformity correction, low rates of infection, and implant-related complications [6,12-14]. Sponseller et al. [6] investigated 32 children who underwent spinal deformity surgery and found that correction of pelvic obliquity $\left(20^{\circ} \pm 11^{\circ}, 70 \%\right)$ was significantly better with SAI screw placement than with other iliac anchors. Elder et al. [12] conducted a retrospective study comparing 25 cases involving iliac screws and 65 cases involving SAI screws, and found that reoperation, surgical-site infection, wound dehiscence, and symptomatic screw prominence were less frequent with SAI screws. Shabtai et al. [14] also reported that in patients with neuromuscular scoliosis, implant failure rate was lower and surgical revision was less frequent with SAI screws than with iliac screws. In other reports, the reoperation rate after iliac screw fixation was as high as $35 \%-48 \%$, and lumbopelvic fixation using SAI screws reduced the need for reoperation by as much as $8.6 \%-14.5 \%[13,15,16]$. Thus, SAI screws appear to have advantages over iliac screws in terms of reducing implant failure and lowering the rate of reoperation.

However, SAI screws have a disadvantage. As the screw crosses the unfused sacroiliac joint, there is an increased potential risk of screw loosening and breakage after fixation [6]. However, few studies have investigated the rate of these screw-related complications [6]. In the current study, loosening was observed in $50 \%$ of the SAI screws at 2 years after surgery, and in approximately half of these cases, screw loosening was present at 6 months after surgery. No studies have investigated the prevalence and timing of SAI screw loosening. Thus, we compared our data with the data from a previous study performed by Banno et al. [11] on iliac screws. The previous study mentioned that loosening was observed in $27.8 \%$ of patients on Xrays and that this screw loosening had already been detected at 6 months after surgery in $80 \%$ of cases. The rate of screw loosening at 2 years was lower in this previous study than in our study; however, the difference in evaluation methods for screw loosening (X-ray, CT) could have greatly affected the results. Additionally, in this previous 
study, the rate of rod breakage around S1 screws was as high as $11.1 \%$, and the rate of pseudoarthrosis was higher than the rate in our study, suggesting that detection of iliac screw loosening on CT could be better than that on $\mathrm{X}$-rays. It is important to note that the rate of screw loosening detected at 6 months was lower in our study (50\%) than in this previous study (80\%). This might be attributable to the high biomechanical stability of SAI screws [17]. Owing to this high stability of SAI screws, screw loosening is more likely to be detected at 1 year, rather than at 6 months, after surgery. Interestingly, in our study, the number of loose SAI screws detected at 1 year did not increase at 2 years after surgery. This finding suggests that once L5-S bony fusion is achieved, the possibility of progressive screw loosening is low, even though the screw penetrates the sacroiliac joint.

SAI screw loosening was associated with pseudoarthrosis at L5-S; therefore, SAI screw loosening was determined to be a clinically important problem. Although only one patient had symptomatic pseudoarthrosis at L5-S necessitating revision surgery in the present study, pseudoarthrosis may manifest if rod breakage occurs in the future [18]. Therefore, careful observation is required in cases without fusion.

Old age, low screw density, large PI, history of revision surgery, failure to restore LL, postoperative sagittal imbalance, insufficient sacropelvic fixation, and other such factors have been reported as risk factors after sacropelvic fixation of screw loosening and pseudoarthrosis [3,9-11]. Kim et al. [9] reported that the rate of S1 screw loosening was $24.4 \%$ in 156 patients who underwent lumbosacral fixation for degenerative lumbar disease, and that the risk factors for screw loosening were age, poor BMD, long fusion ( $\geq 3$ levels), high PI, and high mismatch of PI and LL. Banno et al. [11] investigated the risk factors for iliac screw loosening in 72 patients with adult spinal deformity and concluded that the misplacement of iliac screws and insufficient correction (PI-LL $>10^{\circ}$ ) were significant risk factors for iliac screw loosening. Kim et al. [3] also investigated 144 cases of long adult spinal deformity instrumentation and found that $24 \%$ of the cases showed pseudoarthrosis. Thoracolumbar kyphosis, osteoarthritis of the hip joint, the thoracoabdominal approach, positive sagittal balance $\geq 5 \mathrm{~cm}$, old age ( $\geq 55$ years), and incomplete sacropelvic fixation were found to significantly increase the risk for pseudoarthrosis [3]. In the present study, a low number of SAI screw contact points with the iliac cortical bone and postoperative PI-LL $>10^{\circ}$ were found to be significant risk factors for screw loosening. As mentioned above, PI-LL mismatch has been reported as a risk factor for pseudoarthrosis; thus, appropriate correction of LL is crucial for minimizing screw loosening. In addition, appropriate SAI screw placement with regard to contact with the cortical bone is important. In a previous report [11], misplacement of iliac screws was found to be a risk factor for screw loosening; thus, initial rigid internal fixation secondary to appropriate screw placement is crucial to prevent screw loosening.

For the accurate insertion of SAI screws, a threedimensional (3D) image-guidance system could be useful. Nottimeier et al. [19] reported that of the 32 screws used in 20 patients who underwent lumbosacral fusion with S2 alar screws using this 3D image-guidance technique, five screws penetrated anteriorly without clinical consequences, and they concluded that safe placement of SAI screws was possible with this guidance technique. Although no report has yet investigated the accuracy of SAI screws used in conjunction with a 3D image-guidance system, in the present study only one SAI screw penetrated the iliac cortical bone $(2 \mathrm{~mm})$, suggesting that this imageguidance system can permit safe screw insertion. Additionally, in the present study, a low number of SAI screw contact points with the iliac cortical bone was determined to be a risk factor for SAI screw loosening; thus, an ideal degree of contact with the cortical bone should be carefully considered when SAI screws are inserted. Computer navigation-guided screw insertion techniques, such as the $\mathrm{O}$-arm, allow for screw insertion with intraoperative 3D images of the screw trajectory, making such a technique a safe and reliable method of support [20,21]. A roboticguided SAI screw insertion technique might also show improved accuracy. Bederman et al. [22] investigated robotic guidance for SAI screw insertion in cases of adult spinal deformity and reported acceptable accuracy. In the near future, robotic-guided and computer navigationguided systems will improve, making them even more "surgeon-friendly" and also making them accurate support systems.

The present study had a few limitations. First, this was a retrospectively analyzed study. Second, the study included a relatively small number of cases. Third, the difference in SAI contact points (1.8 versus 2.2) between with and without screw loosening was very small; therefore, further analysis with a greater number of patients is essential 
to validate the results. Lastly, minimum follow-up was 2 years, which is not long enough to evaluate all the nonunions. Further studies at a larger scale conducted over a longer term (over 5 years after surgery) are required to validate our results.

\section{Conclusions}

SAI screw loosening is closely associated with pseudoarthrosis. Female sex, PI-LL mismatch, and a low number of SAI screw contact points with the iliac cortical bone are risk factors for screw loosening. Thus, appropriate screw insertion and optimal LL restoration are crucial.

\section{Conflict of Interest}

Tokumi Kanemura is a consultant of NUVASIVE and Medtronic. No potential conflict of interest relevant to this article was reported.

\section{References}

1. Kebaish KM. Sacropelvic fixation: techniques and complications. Spine (Phila Pa 1976) 2010;35:224551.

2. Guler UO, Cetin E, Yaman O, et al. Sacropelvic fixation in adult spinal deformity (ASD); a very high rate of mechanical failure. Eur Spine J 2015;24:1085-91.

3. Kim YJ, Bridwell KH, Lenke LG, Rhim S, Cheh G. Pseudarthrosis in long adult spinal deformity instrumentation and fusion to the sacrum: prevalence and risk factor analysis of 144 cases. Spine (Phila Pa 1976) 2006;31:2329-36.

4. Alegre GM, Gupta MC, Bay BK, Smith TS, Laubach JE. S1 screw bending moment with posterior spinal instrumentation across the lumbosacral junction after unilateral iliac crest harvest. Spine (Phila Pa 1976) 2001;26:1950-5.

5. Leong JC, Lu WW, Zheng Y, Zhu Q, Zhong S. Comparison of the strengths of lumbosacral fixation achieved with techniques using one and two triangulated sacral screws. Spine (Phila Pa 1976) 1998;23:2289-94.

6. Sponseller PD, Zimmerman RM, Ko PS, et al. Low profile pelvic fixation with the sacral alar iliac technique in the pediatric population improves results at two-year minimum follow-up. Spine (Phila Pa 1976)
2010;35:1887-92.

7. Chang TL, Sponseller PD, Kebaish KM, Fishman EK. Low profile pelvic fixation: anatomic parameters for sacral alar-iliac fixation versus traditional iliac fixation. Spine (Phila Pa 1976) 2009;34:436-40.

8. O’Brien JR, Yu WD, Bhatnagar R, Sponseller P, Kebaish KM. An anatomic study of the S2 iliac technique for lumbopelvic screw placement. Spine (Phila Pa 1976) 2009;34:E439-42.

9. Kim JB, Park SW, Lee YS, Nam TK, Park YS, Kim YB. The effects of spinopelvic parameters and paraspinal muscle degeneration on S1 screw loosening. J Korean Neurosurg Soc 2015;58:357-62.

10. Cho W, Mason JR, Smith JS, et al. Failure of lumbopelvic fixation after long construct fusions in patients with adult spinal deformity: clinical and radiographic risk factors: clinical article. J Neurosurg Spine 2013;19:445-53.

11. Banno T, Hasegawa T, Yamato Y, et al. Prevalence and risk factors of iliac screw loosening after adult spinal deformity surgery. Spine (Phila Pa 1976) 2017;42:E1024-30.

12. Elder BD, Ishida W, Lo SL, et al. Use of S2-alar-iliac screws associated with less complications than iliac screws in adult lumbosacropelvic fixation. Spine (Phila Pa 1976) 2017;42:E142-9.

13. Mazur MD, Ravindra VM, Schmidt MH, et al. Unplanned reoperation after lumbopelvic fixation with S-2 alar-iliac screws or iliac bolts. J Neurosurg Spine 2015;23:67-76.

14. Shabtai L, Andras LM, Portman M, et al. Sacral alar iliac (SAI) screws fail 75\% less frequently than iliac screws in neuromuscular scoliosis. J Pediatr Orthop 2017;37:e470-5.

15. Ilyas H, Place H, Puryear A. A comparison of early clinical and radiographic complications of iliac screw fixation versus S2 alar iliac (S2AI) fixation in the adult and pediatric populations. J Spinal Disord Tech 2015;28:E199-205.

16. Ishida W, Elder BD, Holmes C, et al. S2-alar-iliac screws are associated with lower rate of symptomatic screw prominence than iliac screws: radiographic analysis of minimal distance from screw head to skin. World Neurosurg 2016;93:253-60.

17. Hoernschemeyer DG, Pashuck TD, Pfeiffer FM. Analysis of the s2 alar-iliac screw as compared with the traditional iliac screw: does it increase stabil- 
ity with sacroiliac fixation of the spine? Spine J 2017;17:875-9.

18. Tsuchiya K, Bridwell KH, Kuklo TR, Lenke LG, Baldus C. Minimum 5-year analysis of L5-S1 fusion using sacropelvic fixation (bilateral S1 and iliac screws) for spinal deformity. Spine (Phila Pa 1976) 2006;31:303-8.

19. Nottmeier EW, Pirris SM, Balseiro S, Fenton D. Three-dimensional image-guided placement of S2 alar screws to adjunct or salvage lumbosacral fixation. Spine J 2010;10:595-601.

20. Ishikawa $Y$, Kanemura $T$, Yoshida G, et al. Intraoperative, full-rotation, three-dimensional image (O-arm)- based navigation system for cervical pedicle screw insertion. J Neurosurg Spine 2011;15:472-8.

21. Ishikawa Y, Kanemura T, Yoshida G, Ito Z, Muramoto A, Ohno S. Clinical accuracy of three-dimensional fluoroscopy-based computer-assisted cervical pedicle screw placement: a retrospective comparative study of conventional versus computer-assisted cervical pedicle screw placement. J Neurosurg Spine 2010;13:606-11.

22. Bederman SS, Hahn P, Colin V, Kiester PD, Bhatia NN. Robotic guidance for S2-alar-iliac screws in spinal deformity correction. Clin Spine Surg 2017;30:E49-53. 